\title{
LIPSCHITZ FUNCTIONS AND SPECTRAL SYNTHESIS
}

\author{
SUNG-YUNG LEE
}

Abstract. An $S$-set in the circle group $T$ is a closed subset $S$ of $T$ for which $\overline{j(S)}=k(S)$. We construct a non- $S$-set $S$ satisfying

$$
\bigcup_{\alpha>0} \operatorname{Lip}_{\alpha}(T) \cap k(S) \subset \overline{j(S)} \text {. }
$$

Thus $\operatorname{Lip}_{\alpha}(T) \cap A(T)$ is not a big enough part of $A(T)$ to test the synthesizability of a given closed subset of $T$.

1. Introduction. Let $A(T)$ denote the Banach algebra of functions with absolutely convergent Fourier series. For a closed subset $S$ of $T$, we define

$$
k(S)=\{f \in A(T): f=0 \text { on } S\}
$$

and

$$
j(S)=\{f \in k(S): f=0 \text { on a neighborhood of } S\} .
$$

A closed subset $S$ of $T$ is called an $S$-set if the closure of $j(S)$ in $A(T)$ equals $k(S)$. For notations and basic facts about spectral synthesis, we refer the reader to [1].

It is well known that functions in the Lipschitz $\operatorname{space}_{\operatorname{Lip}}(T)$ are synthesizable, in the sense that

$$
\operatorname{Lip}_{1 / 2}(T) \cap k(S) \subset \overline{j(S)},
$$

for any closed subset $S$ of $T$. On the other hand, for each $\alpha$ less than $\frac{1}{4}$, there are nonsynthesizable functions in $\operatorname{Lip}_{\alpha}(T) \cap A(T)$ (see [2]).

In [6], D. J. Newman shows that, for any closed set $S$ of Lebesgue measure 0 , there is a constant $\alpha>0$ such that

$$
\operatorname{Lip}_{\alpha}(T) \cap k(S) \subset \overline{j(S)} .
$$

Using his condition on $\alpha$, one can see that, for some $S$, the condition (1) holds for every positive $\alpha$, that is,

$$
\left(\bigcup_{\alpha>0} \operatorname{Lip}_{\alpha}(T)\right) \cap k(S) \subset \overline{j(S)}
$$

Since $\operatorname{Lip}_{\alpha}(T) \cap A(T)$, when $0<\alpha<\frac{1}{4}$, is big enough to contain nonsynthesizable functions, one might guess that the condition (2) is strong enough to imply that $S$ is an $S$-set.

Received by the editors November 18, 1980.

1980 Mathematics Subject Classification. Primary 43A45.

Key words and phrases. Spectral synthesis, $S$-sets, Lipschitz function.

(C) 1981 American Mathematical Society 0002-9939/81/0000-0560/\$02.25 
In this note we shall construct (Theorem 1) a non-S-set that satisfies condition (2), disproving the above conjecture. Our method can be generalized to get a stronger result (Theorem 2).

2. Lemmas. In this section, let $S$ denote any closed set of Lebesgue measure 0 . The complement $S^{c}=T-S$ is a countable union of open intervals. Write $S^{c}=\cup_{n=0}^{\infty} I_{n}$, where $\varepsilon_{n}=\left|I_{n}\right|$, the length of $I_{n}$, for $n=0,1,2, \ldots$ Thus $2 \pi=$ $\sum_{n=0}^{\infty} \varepsilon_{n}$.

First of all, we quote a lemma from [6].

LEMMA 1. If $\sum \varepsilon_{n}^{\alpha}\left|\log \varepsilon_{n}\right|<\infty$, then (1) holds.

Note that if $\sum \varepsilon_{n}^{\alpha}<\infty$ for every $\alpha$ then an easy calculation shows that $\sum \varepsilon_{n}^{2 \alpha}\left|\log \varepsilon_{n}\right|<\infty$. Thus (2) holds for $S$. One interesting observation is that, under this condition, condition (2) holds for every closed subset of $S$, as we shall see below.

LEMMA 2. Let $S$ be as above and $S^{\prime}$ be a closed subset. Write $S^{c}=\cup_{n=0}^{\infty} I_{n}$, $\left(S^{\prime}\right)^{c}=\cup_{n=0}^{\infty} J_{n}$, where $\left|I_{n}\right|=\varepsilon_{n}$ and $\left|J_{n}\right|=\varepsilon_{n}^{\prime}$. If $\sum \varepsilon_{n}^{\alpha}<\infty$ and $0<\alpha<1$, then $\Sigma\left(\varepsilon_{n}^{\prime}\right)^{\alpha}<\infty$.

Proof. Notice that, for every sequence $\left\{x_{n}\right\}$ of positive real numbers, $\sum_{n=0}^{\infty} x_{n}^{\alpha}$ $\geqslant\left(\sum x_{n}\right)^{\alpha}$.

Since $S^{\prime} \subset S, S^{c} \subset\left(S^{\prime}\right)^{c}$ so that each $I_{n}$ is contained in exactly one of the $J_{k}$ 's. Now it follows that

$$
\varepsilon_{k}^{\prime}=\left|J_{k}\right|=\left|\left(J_{k} \cap S\right) \cup\left[\bigcup_{n=0}^{\infty}\left(J_{k} \cap I_{n}\right)\right]\right|=\sum_{n=0}^{\infty}\left|J_{k} \cap I_{n}\right|=\sum_{I_{n} \subset J_{k}} \varepsilon_{n} .
$$

Thus

$$
\sum_{n=0}^{\infty} \varepsilon_{n}^{\alpha}=\sum_{k=0}^{\infty}\left(\sum_{I_{n} \subset J_{k}} \varepsilon_{n}^{\alpha}\right)>\sum_{k=0}^{\infty}\left(\sum_{I_{n} \subset J_{k}} \varepsilon_{n}\right)^{\alpha}=\sum_{k=0}^{\infty}\left(\varepsilon_{k}^{\prime}\right)^{\alpha}
$$

We use these lemmas to prove the following lemma.

LEMMA 3. Let $S$ be as above. If $\sum \varepsilon_{n}^{\alpha}<\infty$ for every $\alpha>0$, then every closed subset $S^{\prime}$ of $S$ satisfies (2).

3. Construction. The first part of our construction will be that of Kahane and Salem [4, p. 13], slightly modified to get a set of Lebesgue measure 0.

Take any sequence $\left\{t_{k}\right\}_{k=0}^{\infty}$ of positive real numbers so that $\Sigma_{0}^{\infty} 2^{k} t_{k}<2 \pi$ and $\Sigma_{0}^{\infty} 2^{k} t_{k}^{\alpha}<\infty$ for each $\alpha>0$. For example, one may take $t_{k}=2^{-k^{2}}, k=$ $0,1,2, \ldots$ Then

$$
\sum_{0}^{\infty} 2^{k} 2^{-k^{2}}=1+\sum_{1}^{\infty} 2^{k-k^{2}} \leqslant 1+\sum_{0}^{\infty} 2^{-k}<2 \pi
$$

and $\Sigma_{0}^{\infty} 2^{k}\left(2^{-k^{2}}\right)^{\alpha}=\Sigma_{0}^{\infty} 2^{-\alpha k^{2}+k}<\infty$ by the ratio test.

Put $\varepsilon_{n}=t_{k}$ if $2^{k} \leqslant n<2^{k+1}, k=0,1,2, \ldots$, and $\varepsilon_{0}=2 \pi-\Sigma_{1}^{\infty} \varepsilon_{n}$. 
Let $I_{0}=\left(\Sigma_{1}^{\infty} \varepsilon_{n}, 2 \pi\right)$, and

$$
E_{0}=E_{0}^{1}=\left[0, \sum_{1}^{\infty} \varepsilon_{n}\right]=T-I_{0} .
$$

Note that $\left|I_{0}\right|=2 \pi-\Sigma_{1}^{\infty} \varepsilon_{n}=\varepsilon_{0}$. Let $I_{1}$ be the open interval of length $\varepsilon_{1}$ and having center at $\frac{1}{2} \Sigma_{1}^{\infty} \varepsilon_{n}$, i.e., at the center of the interval $E_{0}^{1}$. Then $E_{0}^{1}-I$ is the union of two closed intervals. Let $E_{1}^{1}$ denote the left interval and $E_{1}^{2}$ the right one. Put $E_{1}=E_{1}^{1} \cup E_{1}^{2}$. Notice that

$$
\left|E_{1}^{1}\right|=\left|E_{1}^{2}\right|=\frac{1}{2} \sum_{2}^{\infty} \varepsilon_{n} \text { and } E_{1} \subset E_{0} .
$$

Suppose we have defined $E_{k}$ as a disjoint union of closed intervals $E_{k}^{1}, \ldots, E_{k}^{2^{k}}$ $\subset E_{k-1}$ so that $\left|E_{k}^{1}\right|=\left|E_{k}^{2}\right|=\cdots=\left|E_{k}^{2^{k}}\right|$. We define $E_{k+1}$ as follows.

Given $j=1,2, \ldots, 2^{k}$, let $I_{2^{k}+j-1}$ be the open interval of length $\varepsilon_{2^{k}+j-1}=t_{k}$ and having the same center as $E_{k}^{j}$. Then $E_{k}^{j}-I_{2^{k}+j-1}$ is the union of two closed intervals of the same length, say $E_{k+1}^{2 j-1}$ and $E_{k+1}^{2 j}$, the left interval and the right one, respectively. Put $E_{k+1}=\cup_{j=1}^{2^{k+1}} E_{k+1}^{j}$. Then $S=\cap_{n=0}^{\infty} E_{k}$ is a perfect set of measure 0. Also, by our choice,

$$
S^{c}=\bigcup_{0}^{\infty} I_{n}, \quad\left|I_{n}\right|=\varepsilon_{n}
$$

and $\sum \varepsilon_{n}^{\alpha}=\sum 2^{k} t_{k}^{\alpha}<\infty$, for each positive $\alpha$.

Next we shall show that $S$ is a "perfect symmetric" set of the type employed in Kahane and Katznelson's paper [3], that is,

$$
S=\left\{r_{0}+\sum_{1}^{\infty} \delta_{k} r_{k}: \delta_{k}= \pm 1\right\}
$$

for some suitable sequence $\left\{r_{k}\right\}$ of real numbers. This fact seems to be well known, but the author could not find its proof.

Put $r_{0}=\frac{1}{2}\left|E_{0}^{1}\right|$, and

$$
r_{k}=\frac{1}{2}\left(\left|E_{k-1}^{1}\right|-\left|E_{k}^{1}\right|\right)
$$

for $k \geqslant 1$. Then $r_{0}$ is the midpoint of $E_{0}^{1}$, and $r_{0}+\sum_{1}^{n} \delta_{k} r_{k}$ is that of some $E_{n}^{j}$, for $n \geqslant 1$.

Indeed, suppose $r_{0}+\sum_{1}^{n} \delta_{k} r_{k}$ is the midpoint of $E_{n}^{j}$. If $\delta_{n+1}=1$, the midpoint of $E_{n+1}^{2 j}$ is $\left(r_{0}+\sum_{1}^{n} \delta_{k} r_{k}\right)+\left(\frac{1}{2}\left|I_{2^{n+1}+j-1}\right|+\frac{1}{2}\left|E_{j+1}^{2 j}\right|\right)$. (Remember that the first term is the midpoint of $E_{n}^{j}$ and that the second is the distance between midpoints of $E_{n}^{j}$ and $E_{n+1}^{2 j}$.) But $\left|E_{n}^{j}\right|=\left|I_{2^{n+1}+j-1}\right|+2\left|E_{n+1}^{2 j}\right|$, so that

$$
\frac{1}{2}\left|I_{2^{n+1}+j-1}\right|+\frac{1}{2}\left|E_{n+1}^{2 j}\right|=\frac{1}{2}\left(\left|E_{n}^{j}\right|-\left|E_{n+1}^{2 j}\right|\right)=r_{k} .
$$

Hence the midpoint of $E_{n+1}^{2 j}$ is $r_{0}+\sum_{1}^{n+1} \delta_{k} r_{k}$. Similarly, if $\delta_{n+1}=-1$, then $r_{0}+\sum_{1}^{n+1} \delta_{k} r_{k}$ is the midpoint of $E_{n+1}^{2 j-1}$.

Given any $r_{0}+\sum_{1}^{\infty} \delta_{k} r_{k}$ and $n>1, r_{0}+\sum_{1}^{n} \delta_{k} r_{k}$ is the midpoint of some $E_{n}^{j}$, and

$$
\left|\sum_{k=n+1}^{\infty} \delta_{k} r_{k}\right| \leqslant \sum_{n+1}^{\infty} r_{k}=\frac{1}{2} \sum_{n+1}^{\infty}\left(\left|E_{k-1}^{1}\right|-\left|E_{k}^{1}\right|\right)=\frac{1}{2}\left|E_{n}^{1}\right|=\frac{1}{2}\left|E_{n}^{j}\right| \text {. }
$$


Thus $r_{0}+\sum_{1}^{\infty} \delta_{k} r_{k}=\left(r_{0}+\sum_{1}^{n} \delta_{k} r_{k}\right)+\sum_{n+1}^{\infty} \delta_{k} r_{k} \in E_{n}^{j} \subset E_{n}$ and hence

$$
r_{0}+\sum_{1}^{\infty} \delta_{k} r_{k} \in S
$$

Conversely, let $x \in S$ be arbitrary. For $n>1$, since $x \in E_{n}, x \in E_{n}^{j_{n}}$ for some $j_{n}$. But $E_{n}^{j_{n}}$ contains only $E_{n+1}^{2 j_{n}-1}$ and $E_{n+1}^{2 j_{n}}$. Thus $j_{n+1}$ is either $2 j_{n}-1$ or $2 j_{n}$. Define

$$
\delta_{n}= \begin{cases}-1, & \text { if } j_{n+1}=2 j_{n}-1, \\ 1, & \text { if } j_{n+1}=2 j_{n} .\end{cases}
$$

Now, by using induction once more, one can see that $r_{0}+\sum_{1}^{n} \delta_{k} r_{k}$ is the midpoint of $E_{n}^{j_{n}}$. So it follows that

$$
\left|x-\left(r_{0}+\sum_{1}^{n} \delta_{k} r_{k}\right)\right|<\frac{1}{2}\left|E_{n}^{j_{n}}\right|=\frac{1}{2}\left|E_{n}^{1}\right| \rightarrow 0,
$$

as $n \rightarrow \infty$. Thus it follows that $x=r_{0}+\Sigma_{1}^{\infty} \delta_{k} r_{k}$, which proves our claim.

Since no set of the form $\left\{r_{0}+\sum_{1}^{\infty} \delta_{k} r_{k}: \delta_{k}= \pm 1\right\}$ is a set of spectral resolution [3], $S$ contains a closed subset $S^{\prime}$ which is not an $S$-set. But, by Lemma 3, the condition (2) holds for $S^{\prime}$. If we summarize these results, we get

THEOREM 1. There is a non-S-set $S$, satisfying

$$
\left(\bigcup_{\alpha>0} \operatorname{Lip}_{\alpha}(T)\right) \cap k(S) \subset \overline{j(S)}
$$

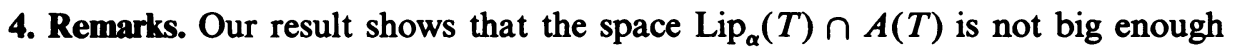
to test the synthesizability of a given closed set.

Let $\varphi$ be any nondecreasing continuous function on $[0,2 \pi]$ satisfying the following conditions:

$\varphi(0)=0, \varphi(x)>0$ if $x>0, \varphi(x) / x$ is decreasing on $(0,2 \pi]$ and $\varphi(x+y)<$ $\varphi(x)+\varphi(y)$ for positive $x$ and $y$.

Let $V_{\varphi}=\left\{f \in C(T):\left\|f_{\tau}-f\right\|_{C(T)} \leqslant C \varphi(|\tau|)\right.$, for some $\left.C>0\right\}$, where $f_{\tau}(t)=$ $f(t-\tau)$ is the translation of $f$ by $\tau$. Notice that $\operatorname{Lip}_{\alpha}(T)=V_{\varphi}$ for $\varphi(t)=t^{\alpha}$.

If we use Theorem 3 of [6] instead of Lemma 1, we can modify our proofs to get the following theorem, which says, roughly, that the space of functions satisfying some regularity condition is not big enough to test the synthesizability of a set.

TheOREM 2. Let $\varphi$ be as above. Then there is a non-S-set $S$, satisfying

$$
V_{\varphi} \cap k(S) \subseteq \overline{j(S)} .
$$

On p. 88 of [5], T. W. Körner constructed, for every continuous increasing function $H$ on $[0, \infty)$ with $H(0)=0$, a non- $S$-set $S$ having, along with other properties, Hausdorff $H$-measure 0 . If we take the function $H(t)=-1 / \log t$, $0<t<\frac{1}{3}$, we get $t^{\alpha}=o(H(t))$ as $t \rightarrow 0, \alpha>0$, and hence the Hausdorff $\alpha$ measure $H^{\alpha}(S)=0$ (see [4, p. 26]). Thus, the Hausdorff dimension, $\alpha(S)$, of $S$ is 0 , so that $S$ satisfies the condition (2) (see [6]). This gives an alternate proof of our Theorem 1.

The author wishes to thank his advisor, Professor C. R. Warner, for his encouragement and help. 


\section{REFERENCES}

1. J. Benedetto, Spectral synthesis, Teubner, Stuttgart, 1975.

2. J. P. Kahane, Sur le théorème de Beurling-Pollard, Math. Scand. 21 (1967), 71-79.

3. J. P. Kahane and Y. Katznelson, Contribution à deux problèmes, concernant les fonctions de la classe A, Israel J. Math. 1 (1963), 110-131.

4. J. P. Kahane and R. Salem, Ensembles parfaits et séries trigonométriques, Hermann, Paris, 1963.

5. T. W. Körner, A pseudofunction on a Helson set. I, Astérisque 5 (1973), 3-224.

6. D. J. Newman, Some results in spectral synthesis, Duke Math. J. 27 (1960), 359-361.

Department of Mathematics, University of Maryland, College Park, Maryland 20742 